\title{
Article \\ A New Tumor Burden Score and Albumin-Bilirubin Grade-Based Prognostic Model for Hepatocellular Carcinoma
}

\author{
Shu-Yein Ho ${ }^{1,2,3}$, Po-Hong Liu ${ }^{3,4}$, Chia-Yang Hsu ${ }^{3,5}$, Yi-Hsiang Huang ${ }^{3,6,7} \mathbb{C}$, Jia-I Liao ${ }^{3,6}$, Chien-Wei Su ${ }^{3,6} \mathbb{D}^{\text {, }}$ \\ Ming-Chih Hou ${ }^{3,6}$ and Teh-Ia Huo $2,3,8, * \mathbb{C}$
}

1 Division of Gastroenterology and Hepatology, Min-Sheng General Hospital, Taoyuan 330, Taiwan; zawzaw222@gmail.com

2 Department of Medical Research, Taipei Veterans General Hospital, Taipei 11217, Taiwan

3 School of Medicine, National Yang Ming Chiao Tung University, Taipei 11217, Taiwan; stuartliu@gmail.com (P.-H.L.); chiayanghsu2@gmail.com (C.-Y.H.); yhhuang@vghtpe.gov.tw (Y.-H.H.); alohawhs@hotmail.com (J.-I.L.); cwsu2@vghtpe.gov.tw (C.-W.S.); mchou@vghtpe.gov.tw (M.-C.H.)

4 Department of Internal Medicine, University of Texas Southwestern Medical Center, Dallas, TX 75390, USA

5 Veterans Affairs Sierra Nevada Healthcare System, Reno, NV 89502, USA

6 Division of Gastroenterology and Hepatology, Department of Medicine, Taipei Veterans General Hospital, Taipei 11217, Taiwan

7 Institute of Clinical Medicine, National Yang Ming Chiao Tung University, Taipei 11217, Taiwan

8 Institute of Pharmacology, National Yang Ming Chiao Tung University, Taipei 11217, Taiwan

* Correspondence: tihuo@vghtpe.gov.tw; Tel.: +886-2-2871-2121; Fax: +886-2-2873-9318

Citation: Ho, S.-Y.; Liu, P.-H.; Hsu, C.-Y.; Huang, Y.-H.; Liao, J.-I.; Su, C.-W.; Hou, M.-C.; Huo, T.-I. A New Tumor Burden Score and AlbuminBilirubin Grade-Based Prognostic Model for Hepatocellular Carcinoma. Cancers 2022, 14, 649. https:// doi.org/10.3390/cancers14030649

Academic Editor: Dimitrios Moris

Received: 17 December 2021

Accepted: 24 January 2022

Published: 27 January 2022

Publisher's Note: MDPI stays neutral with regard to jurisdictional claims in published maps and institutional affiliations.

Copyright: (C) 2022 by the authors. Licensee MDPI, Basel, Switzerland. This article is an open access article distributed under the terms and conditions of the Creative Commons Attribution (CC BY) license (https:// creativecommons.org/licenses/by/ $4.0 /)$.
Simple Summary: The survival of patients with hepatocellular carcinoma (HCC) is highly variables, due to heterogeneous tumor burden and liver dysfunction. Tumor burden score (TBS) is a continuous variable to measure the extent of tumor involvement, and the albumin-bilirubin (ALBI) grade is an objective model to estimate hepatic functional reserve. Six prognostic predictors-including TBS, ALBI grade, ascites, serum $\alpha$-fetoprotein level, vascular invasion or distant metastasis, and performance status-were linked with survival in a multivariate Cox model. We used these predictors to establish a new prognostic model-the TBS-ALBI system - to predict patient outcomes. Significant survival differences were found in different TBS-ALBI scores in the derivation and validation cohorts. This new system can also discriminate survival differences in patients with different viral etiologies, cancer stages, and treatment modalities. This study shows that the TBS-ALBI system is a feasible and user-friendly prognostic model for HCC.

\begin{abstract}
The prognosis of hepatocellular carcinoma (HCC) varies widely due to variable tumor extent and liver reserve. We aimed to develop and validate a new prognostic model based on tumor burden score (TBS) and albumin-bilirubin (ALBI) grade for HCC. We prospectively identified 3794 HCC patients who were randomized into derivation and validation groups. Survival predictors were evaluated by a multivariate Cox model. The TBS-ALBI system allocated two points for high TBS and ALBI grade 3, and one point each for the presence of ascites, serum $\alpha$-fetoprotein $\geq 400 \mathrm{ng} / \mathrm{mL}$, vascular invasion or distant metastasis, performance status $2-4$, medium TBS, and ALBI grade 2 , with a maximal score of 8 points. Significant survival differences were found across different TBS-ALBI score groups in the validation cohort (all $p<0.001$ ). The TBS-ALBI system had the lowest corrected Akaike information criterion (AICc) and the highest homogeneity compared with other proposed staging models. The discriminative ability of the TBS-ALBI system was consistently stable across different viral etiologies, cancer stages, and treatment strategies. Conclusions: This new TBS-ALBI system is a feasible and robust prognostic system in comparison with other systems; it is a userfriendly tool for long-term outcome assessment independent of treatment modality and cancer stage in HCC.
\end{abstract}

Keywords: tumor burden score; albumin-bilirubin grade; hepatocellular carcinoma; prognosis 


\section{Introduction}

Hepatocellular carcinoma (HCC) is a highly prevalent liver cancer, and was the fourth most common cause of cancer-related death in 2018 globally [1]. The major etiologies of $\mathrm{HCC}$ are chronic hepatitis $\mathrm{B}$ and $\mathrm{C}$ virus (HBV, HCV) infection, alcoholism, and nonalcoholic fatty liver disease (NAFLD) [2]. Surgery, liver transplant, and local ablation are primarily indicated for early-stage HCC [2,3]. For those belonging with intermediate and advanced disease, transarterial chemoembolization (TACE) and systemic therapies, such as targeted therapy or immunotherapy, are usually recommended $[4,5]$.

Cancer staging plays a crucial role in the management of HCC [6]. The Barcelona Clinic Liver Cancer (BCLC) system has been widely used for prognostic prediction and treatment guidance, and is endorsed by current HCC practice guidelines [2,3]. The reported survival predictors include tumor burden, severity of liver dysfunction, and performance status. Notably, tumor diameter and nodules indicate the extent of the tumor in HCC, and are included in the BCLC and Hong Kong Liver Cancer (HKLC) staging systems. These two parameters act in a dichotomous fashion with arbitrary cutoffs. The Milan criteria and the Up-to-7 criteria have also been used to evaluate the extent of tumors in HCC $[7,8]$. Still, these two models have limitations due to their categorical allocation, and it could be quite difficult to evaluate the prognosis for patients with variable tumor size and nodules. Investigators suggested the use of continuous tumor diameter and nodules to represent tumor burden. Mazzaferro et al. proposed the concept of a "metro-ticket model": an increase in the diameter of the tumor and/or number of lesions and the decrease in patient survival [9]. Alternatively, continuous variables—-such as total tumor diameter (TTD) and total tumor volume (TTV) - have been suggested to assess tumor extent in HCC, but these two models still harbor inevitable shortcomings $[10,11]$. Recently, Sasaki et al. proposed the tumor burden score (TBS), which includes tumor diameter and number of nodules as continuous variables to estimate outcomes in colorectal liver metastasis [12]. TBS was later applied to discriminate survival in HCC patients receiving partial hepatectomy and liver transplant $[13,14]$. The Italian Liver Cancer (ITA.LI.CA) group also used TBS to stratify prognosis in HCC patients undergoing different curative and non-curative modalities [15], indicating that TBS could be a reliable surrogate indicator of tumor burden.

The severity of liver injury is an important predictor in treating HCC. The ChildTurcotte-Pugh (CTP) classification is used to indicate the severity of cirrhosis in the BCLC, HKLC, Cancer of the Liver Italian Program (CLIP), Taipei Integrated Scoring System (TIS), and Japan Integrated System (JIS) staging systems. However, the CTP classification has potential drawbacks, owing to its subjective variables with arbitrarily defined cutoffs [16]. The model for end-stage liver disease (MELD) score has also been used to assess liver injury [17]. More recently, the albumin-bilirubin (ALBI) grade-a simple and objective method based solely on serum levels of albumin and bilirubin-was proposed to evaluate liver dysfunction, and has been validated by several research groups [18-22]. Although many HCC staging systems-including BCLC, HKLC, TIS, JIS, CLIP, Okuda, Tokyo, and tumor-node-metastasis (TNM)-have been used to predict the outcome of HCC [6], the optimal staging system remains unclear. In this study, we aimed to establish a new prognostic model based on TBS and ALBI grade (the TBS-ALBI system) for HCC, and its performance was comprehensively compared with other currently used staging systems.

\section{Materials and Methods}

\subsection{Patient Characteristics}

Between 2002 and 2017, we prospectively identified 3794 HCC patients in Taipei Veterans General Hospital, who were retrospectively analyzed in this study. At diagnosis, patients' demographic data, tumor extent, severity of liver dysfunction, tumor staging, and treatments were recorded; their survival status was inspected every 3-4 months during the disease course until death or cessation of follow-up. Patients with a confirmed diagnosis were discussed in the multidisciplinary cancer board for treatment recommendations. Surgical resection, local ablation therapy, and TACE were employed as previously 
reported [23-25]. Patients were randomly assigned to derivation $(n=1898)$ and validation cohorts $(n=1896)$ at a $1: 1$ ratio for the construction and validation of the new model. This study was approved by the Institutional Review Board of Taipei Veterans General Hospital, and complies with the standards of the Declaration of Helsinki and current ethical guidelines.

\subsection{Definition and Diagnosis}

HCC was diagnosed according to the European Association of the Study of Liver (EASL) and American Association for the Study of Liver Diseases (AASLD) HCC practice guidelines [2,3]. Vascular invasion was defined as tumor invasion of portal veins or their branches, hepatic veins, or large vasculatures such as the inferior vena cava [26]. CT or MRI was performed to detect possible distant metastasis, as previously described [27,28]. Surgical resection, local ablation therapy, and liver transplantation were classified as curative treatments; TACE, systemic therapy, and best supportive care were defined as non-curative treatments.

\subsection{Calculation of TBS}

TBS was defined as the distance from the origin on a Cartesian plane that incorporated two variables: maximum tumor size, and number of liver lesions. The TBS was calculated by using the following equation: $\mathrm{TBS}^{2}=$ (maximum tumor diameter $^{2}+$ (number of tumors $)^{2}$. TBS was divided into 3 groups-low (<3.36), medium (3.36 to 13.74), and high TBS (>13.74)—as described previously [12,13].

\subsection{Calculation of ALBI Score}

The equation for ALBI score is as follows: ALBI score $=\left(0.66 \times \log _{10}\right.$ bilirubin $(\mu \mathrm{mol} / \mathrm{L})$ $-(0.085 \times$ albumin $(\mathrm{g} / \mathrm{L}))$. The cutoff values of ALBI grade $1 / 2$ and grade $2 / 3$ were -2.60 and -1.39 , respectively [18].

\subsection{Development and Validation of the New Prognostic Model}

The new prognostic model was constructed according to the following criteria: (1) the new method contains only clinically available parameters, and (2) it should be easy to use and calculate. The model was investigated in the derivation cohort with parameters including baseline characteristics, extent of tumor involvement, hepatic functional reserve, and performance status. Significant predictors in univariate survival analysis were evaluated by the multivariate Cox proportional hazards model to identify independent predictors. The derived predictors were employed to construct the new model in the derivation cohort. A new prediction score was proposed, giving ordinal scores $(0,1$, and 2$)$ to each of the independent predictors according to the calculated regression coefficients in the statistical model. This newly proposed TBS-ALBI system was established by adding each point of the predictors, and justified in the validation cohort according to differences in BCLC stage, treatment, and etiology of liver disease.

\subsection{Statistics}

We used IBM SPSS Statistics for Windows, version 21.0 (IBM Corp., Armonk, NY, USA), for statistical analysis. The comparison of continuous data and categorical data was carried out via Mann-Whitney U test and the chi-squared or Fisher's exact test, respectively. Kaplan-Meier analysis was used to evaluate the survival differences in cancer patients. A multivariate Cox hazards model was applied to identify prognostic factors. The corrected Akaike information criteria (AICc) were obtained to reveal how staging systems were correlated with survival. The lower the AICc value, the more accurate and informative the model in terms of survival prediction [29]. A $p$-value $<0.05$ was considered statistically significant. 


\section{Results}

\subsection{Baseline Characteristics}

A prospective cohort of 3794 HCC patients was enrolled; their baseline characteristics are shown in Table 1 . The mean age was 66 years, and most $(76 \%)$ patients were male. HBV $(40 \%)$ and HCV (22\%) were the main etiological drivers of HCC. The median TBS was 5.0, and $31 \%$ and $61 \%$ of patients had low TBS and medium TBS, respectively. Vascular invasion or distant metastasis was found in $27 \%$ of patients, and $29 \%$ had serum $\alpha$-fetoprotein (AFP) levels $\geq 400 \mathrm{ng} / \mathrm{mL}$. CTP class A was noted in $73 \%$ of patients, and $38 \%$ and $52 \%$ of patients had ALBI grade 1 and grade 2, respectively. Fifty-two percent of patients received curative treatments. The distribution of patients according to different cancer staging systems is shown in Table 1. There were no significant baseline differences between the derivation and validation cohorts (all $p>0.05$, Table 1 ). TBS was weakly yet significantly and positively correlated with ALBI score (correlation coefficient $=0.206, p<0.001$; Figure 1 ).

Table 1. Baseline demographics $(n=3794)$.

\begin{tabular}{|c|c|c|c|c|}
\hline Variables & All Patients & $\begin{array}{c}\text { Derivation } \\
\text { Cohort }(n=1898)\end{array}$ & $\begin{array}{c}\text { Validation } \\
\text { Cohort }(n=1896)\end{array}$ & $p$ \\
\hline Age (years, mean \pm SD) & $66 \pm 13$ & $64 \pm 13$ & $65 \pm 13$ & 0.715 \\
\hline Male, $n(\%)$ & $2895(76)$ & $1450(76)$ & $1445(75)$ & 0.909 \\
\hline Etiologies of liver disease & & & & 0.894 \\
\hline $\mathrm{HBV}, n(\%)$ & $1513(40)$ & $737(39)$ & $776(41)$ & \\
\hline $\mathrm{HCV}, n(\%)$ & $824(22)$ & $450(24)$ & $374(20)$ & \\
\hline $\mathrm{HBV}+\mathrm{HCV}, n(\%)$ & $135(3)$ & $61(3)$ & $74(4)$ & \\
\hline Others, $n(\%)$ & $1322(35)$ & $650(34)$ & $672(35)$ & \\
\hline $\begin{array}{c}\text { Performance status }(0 / 1 / 2 / 3-4) \text {, } \\
n(\%)\end{array}$ & $\begin{array}{c}2226 / 780 / 431 / 357 \\
(59 / 21 / 11 / 9)\end{array}$ & $\begin{array}{c}1119 / 376 / 218 / 185 \\
(59 / 20 / 12 / 9)\end{array}$ & $\begin{array}{c}1107 / 404 / 213 / 172 \\
(59 / 21 / 11 / 9)\end{array}$ & 0.758 \\
\hline Diabetes mellitus, $n(\%)$ & $972(26)$ & $459(25)$ & $503(27)$ & 0.206 \\
\hline $\begin{array}{l}\text { Tumor nodules } \\
\text { (single/multiple), (\%) }\end{array}$ & $2437 / 1357(64 / 36)$ & $1227 / 671(65 / 35)$ & $1210 / 686(64 / 36)$ & 0.611 \\
\hline $\begin{array}{l}\text { Maximal tumor diameter } \\
\quad \geq 5 \mathrm{~cm}, n(\%)\end{array}$ & $1668(44)$ & $838(44)$ & $830(44)$ & 0.819 \\
\hline Tumor burden score (mean \pm SD) & $5.0 \pm 4.4$ & $6.5 \pm 4.3$ & $6.5 \pm 4.4$ & 0.550 \\
\hline Tumor burden score (low/medium/high) & $1160 / 2299 / 335(31 / 61 / 8)$ & $578 / 1157 / 163(30 / 61 / 9)$ & $582 / 1142 / 172(31 / 60 / 9)$ & 0.900 \\
\hline Vascular invasion or distant metastasis, $n(\%)$ & $1038(27)$ & $494(26)$ & $544(28)$ & 0.069 \\
\hline Serum AFP $\geq 400 \mathrm{ng} / \mathrm{mL}, n(\%)$ & $1117(29)$ & $546(29)$ & $571(30)$ & 0.373 \\
\hline Ascites, $n(\%)$ & $861(23)$ & $432(23)$ & $429(23)$ & 0.938 \\
\hline \multicolumn{5}{|l|}{ Laboratory values (mean $\pm \mathrm{SD}$ ) } \\
\hline Albumin (g/L) & $37 \pm 6$ & $36 \pm 6$ & $36 \pm 6$ & 0.600 \\
\hline Total bilirubin $(\mu \mathrm{mol} / \mathrm{L})$ & $15 \pm 48$ & $26 \pm 51$ & $26 \pm 44$ & 0.685 \\
\hline Platelets $(1000 / \mu \mathrm{L})$ & $153 \pm 96$ & $170 \pm 95$ & $170 \pm 97$ & 0.938 \\
\hline INR of prothrombin time & $1.06 \pm 0.2$ & $1.1 \pm 0.3$ & $1.1 \pm 0.2$ & 0.187 \\
\hline Creatinine $(\mathrm{mg} / \mathrm{dL})$ & $1.0 \pm 1.0$ & $1.2 \pm 1.0$ & $1.2 \pm 1.0$ & 0.944 \\
\hline CTP class $(\mathrm{A} / \mathrm{B} / \mathrm{C})$ & $2787 / 831 / 176(73 / 22 / 5)$ & $1480 / 386 / 104(74 / 20 / 6)$ & $1379 / 445 / 72(72 / 24 / 4)$ & 0.006 \\
\hline CTP score, mean \pm SD & $5.0 \pm 1.5$ & $6.0 \pm 1.5$ & $6.0 \pm 1.5$ & 0.644 \\
\hline ALBI grade $(1 / 2 / 3), n(\%)$ & $1444 / 1970 / 380(38 / 52 / 10)$ & $745 / 943 / 210(39 / 50 / 11)$ & $699 / 1027 / 170(37 / 54 / 9)$ & 0.899 \\
\hline $\begin{array}{l}\text { ALBI score, mean } \pm \text { SD } \\
\text { Tumor staging, }(\%)\end{array}$ & $-2.40 \pm 0.65$ & $-2.29 \pm 0.66$ & $-2.30 \pm 0.63$ & 0.810 \\
\hline BCLC stage $(0 / \mathrm{A} / \mathrm{B} / \mathrm{C} / \mathrm{D})$ & $8 / 24 / 17 / 40 / 11$ & $8 / 24 / 18 / 38 / 11$ & $8 / 25 / 16 / 41 / 11$ & 0.754 \\
\hline HKLC (I/II/III/IV/V) & $32 / 27 / 10 / 8 / 22$ & $32 / 27 / 10 / 8 / 23$ & $32 / 27 / 10 / 9 / 22$ & 0.885 \\
\hline TIS $(0 / 1 / 2 / 3 / 4 / 5 / 6)$ & $36 / 22 / 21 / 12 / 12 / 11 / 6 / 1$ & $37 / 21 / 13 / 11 / 12 / 5 / 1$ & $36 / 22 / 12 / 12 / 11 / 6 / 1$ & 0.378 \\
\hline JIS $(0 / 1 / 2 / 3 / 4 / 5)$ & $9 / 33 / 30 / 17 / 9 / 2$ & $9 / 33 / 30 / 17 / 9 / 2$ & $9 / 33 / 30 / 17 / 9 / 1$ & 0.827 \\
\hline $\operatorname{CLIP}(0 / 1 / 2 / 3 / 4 / 5 / 6)$ & $32 / 26 / 15 / 12 / 9 / 5 / 1$ & $33 / 26 / 15 / 12 / 9 / 4 / 1$ & $31 / 27 / 15 / 11 / 10 / 5 / 1$ & 0.346 \\
\hline Okuda $(1 / 2 / 3)$ & $53 / 38 / 9$ & $53 / 38 / 9$ & $53 / 39 / 8$ & 0.616 \\
\hline Tokyo $(0 / 1 / 2 / 3 / 4 / 5 / 6 / 7 / 8)$ & $6 / 22 / 26 / 19 / 12 / 8 / 4 / 2 / 1$ & $6 / 23 / 25 / 19 / 12 / 8 / 4 / 2 / 1$ & $6 / 21 / 27 / 19 / 13 / 8 / 3 / 2 / 1$ & 0.833 \\
\hline TNM (I/II/III/IV) & $33 / 25 / 36 / 6$ & $34 / 24 / 36 / 6$ & $33 / 24 / 36 / 6$ & 0.681 \\
\hline Treatments, $n(\%)$ & & & & 0.775 \\
\hline Surgical resection & $1107(29)$ & $569(30)$ & $538(28)$ & \\
\hline Local ablation therapy & $680(18)$ & $327(17)$ & $353(19)$ & \\
\hline TACE & $1034(27)$ & $521(27)$ & $513(27)$ & \\
\hline Liver transplantation & $20(1)$ & $9(1)$ & $11(1)$ & \\
\hline Targeted therapy & $303(8)$ & $154(8)$ & $149(8)$ & \\
\hline Others & $650(17)$ & $318(17)$ & $332(17)$ & \\
\hline
\end{tabular}

ALBI: albumin-bilirubin; AFP: alpha-fetoprotein; BCLC: Barcelona Clinic Liver Cancer; CTP: Child-Turcotte-Pugh score; HBV: hepatitis B virus; $\mathrm{HCV}$ : hepatitis $\mathrm{C}$ virus; SD: standard deviation; TACE, transarterial chemoembolization. 


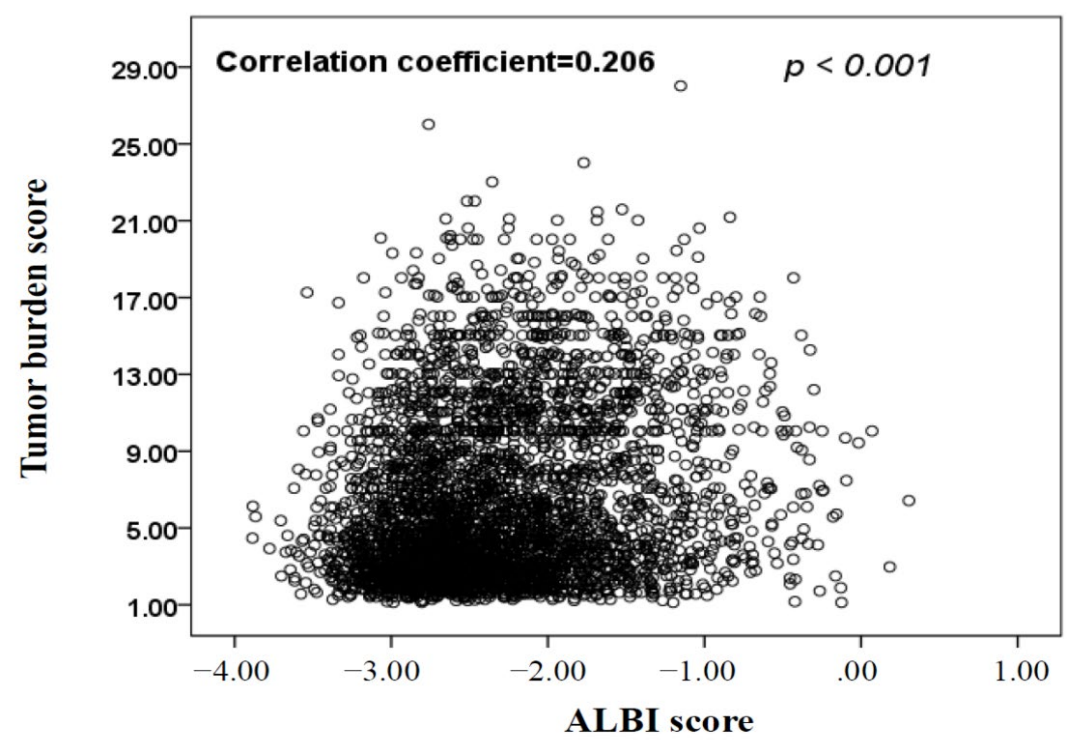

Figure 1. The correlation between tumor burden score (TBS) and albumin-bilirubin (ALBI) score. There was weak but significant correlation between TBS and ALBI score (correlation coefficient = $0.206, p<0.001 ; n=3794)$.

\subsection{Development of the New TBS-ALBI Prognostic System}

In univariate analysis, presence of ascites, serum AFP $\geq 400 \mathrm{ng} / \mathrm{mL}$, vascular invasion or distant metastasis, performance status 2-4, TBS, and ALBI grade were significant variables associated with survival. In the multivariate Cox model, presence of ascites (HR: 1.343, 95\% CI: 1.169-1.542, $p<0.001)$, AFP $\geq 400$ ng/mL (HR: 1.523, 95\% CI: 1.345-1.726, $p<0.001$ ), vascular invasion or distant metastasis (HR: 2.462, 95\% CI: 2.126-2.851, $p<0.001$ ), performance status 2-4 (HR: 1.853, 95\% CI: 1.612-2.129, $p<0.001$ ), medium TBS (HR: 1.655, 95\% CI: 1.446-1.893, $p<0.001$ ), high TBS (HR: 2.395, 95\% CI: 1.919-2.990, $p<0.001$ ), ALBI grade 2 (HR: 1.879, 95\% CI: 1.661-2.126, $p<0.001$ ), and ALBI grade 3 (HR: 3.090, 95\% CI: 2.540-3.761, $p<0.001$ ) were independent predictors of increased mortality (Table 2).

Table 2. Univariate and multivariate analysis of overall survival in HCC patients in the derivation cohort $(n=1898)$.

\begin{tabular}{|c|c|c|c|c|c|c|c|}
\hline \multirow[b]{2}{*}{ Variables } & \multirow[b]{2}{*}{ Number } & \multicolumn{3}{|c|}{ Univariate Analysis } & \multicolumn{3}{|c|}{ Multivariate Analysis } \\
\hline & & $\begin{array}{c}\text { 3-Year } \\
\text { Survival (\%) }\end{array}$ & $\begin{array}{c}\text { 5-Year } \\
\text { Survival }(\%)\end{array}$ & $p$ & $\begin{array}{l}\text { Hazard } \\
\text { Ratio }\end{array}$ & $95 \% \mathrm{CI}$ & $p$ \\
\hline Age $(<55 / \geq 55$ years $)$ & $955 / 943$ & $44 / 47$ & $37 / 34$ & 0.936 & & & \\
\hline Sex (male/female) & $2895 / 899$ & $64 / 73$ & $45 / 52$ & 0.004 & & & \\
\hline HBV (negative/positive) & $905 / 993$ & $45 / 48$ & $31 / 38$ & 0.041 & & & \\
\hline $\mathrm{HCV}$ (negative/positive) & $1311 / 587$ & $46 / 48$ & $35 / 35$ & 0.462 & & & \\
\hline Platelet $(<150,000 / \geq 150,000 / \mu \mathrm{L})$ & $986 / 912$ & $43 / 50$ & $17 / 13$ & 0.034 & & & \\
\hline Ascites (absent/present) & $1466 / 432$ & $55 / 18$ & $41 / 13$ & $<0.001$ & 1.343 & $1.169-1.542$ & $<0.001$ \\
\hline Serum AFP $(<400 / \geq 400 \mathrm{ng} / \mathrm{mL})$ & $1352 / 546$ & $56 / 22$ & $43 / 15$ & $<0.001$ & 1.523 & $1.345-1.726$ & $<0.001$ \\
\hline $\begin{array}{l}\text { Vascular invasion or distant } \\
\text { metastasis (no/yes) }\end{array}$ & $1404 / 494$ & $57 / 9$ & $43 / 6$ & $<0.001$ & 2.312 & $2.010-2.658$ & $<0.001$ \\
\hline Diabetes mellitus (no/yes) & $1429 / 469$ & $48 / 43$ & $36 / 31$ & 0.025 & & & \\
\hline $\begin{array}{l}\text { Performance status } 0-1 / 2-4 \\
\text { Tumor burden score }\end{array}$ & $1119 / 709$ & $54 / 18$ & $42 / 9$ & $<0.001$ & 1.853 & $1.612-2.129$ & $<0.001$ \\
\hline Low & 578 & 71 & 56 & & & & \\
\hline Medium & 1157 & 39 & 28 & $<0.001$ & 1.655 & $1.446-1.893$ & $<0.001$ \\
\hline $\begin{array}{l}\text { High } \\
\text { ALBI }\end{array}$ & 163 & 9 & 6 & $<0.001$ & 2.395 & $1.919-2.990$ & $<0.001$ \\
\hline Grade 1 & 745 & 68 & 55 & & & & \\
\hline Grade 2 & 943 & 37 & 25 & $<0.001$ & 1.879 & $1.661-2.126$ & $<0.001$ \\
\hline Grade 3 & 210 & 11 & 7 & $<0.001$ & 3.090 & $2.540-3.761$ & $<0.001$ \\
\hline
\end{tabular}

HBV: hepatitis B virus; HCV: hepatitis C virus; AFP: $\alpha$-fetoprotein; ALBI: albumin-bilirubin. 
The TBS-ALBI system was developed based on the six predictors from the multivariate model, and the weight score of each variable was based on the predicted risk model. In this new TBS-ALBI system, two points are given for high TBS and ALBI grade 3, and one point is given for medium TBS, ALBI grade 2, presence of vascular invasion, presence of ascites, AFP $\geq 400 \mathrm{ng} / \mathrm{mL}$, and performance status $2-4$; the total score ranges from 0 to 8 points accordingly (Table 3 ).

Table 3. The TBS-ALBI system.

\begin{tabular}{cccc}
\hline Prognostic Factors & \multicolumn{2}{c}{ Score } \\
\hline & $\mathbf{0}$ & $\mathbf{1}$ & $\mathbf{2}$ \\
\hline Tumor burden score & Low & Medium & High \\
ALBI grade & 1 & 2 & 3 \\
Vascular invasion or distant metastasis & Absent & Present & \\
Ascites & Absent & $\geq 400$ & \\
Serum AFP (ng/mL) & $<400$ & $2-4$ \\
Performance status & $0-1$ & & \\
\hline
\end{tabular}

\subsection{Patient Survival in the Derivation Cohort Based on the TBS-ALBI System}

In this 7967 person-year study, the median overall survival was 125 (95\% CI: 102-148) months, 70 (95\% CI: 61-79) months, 36 (95\% CI: 30-41) months, 16 (95\% CI: 13-19) months, 5 (95\% CI: 3.5-6.5) months, and 2 (95\% CI: 1.7-2.3) months for TBS-ALBI scores of 0, 1, 2, 3, 4 , and 5-8 points, respectively. The 1-, 3-, and 5-year survival rates were $99 \%, 85 \%$, and $73 \%$ for TBS-ALBI score $0,92 \%, 71 \%$, and $65 \%$ for score $1,76 \%, 50 \%$, and $30 \%$ for score $2,58 \%$, $31 \%$, and $22 \%$ for score $3,32 \%, 12 \%$, and $6 \%$ for score 4 , and $9 \%, 3 \%$, and $3 \%$ for scores of $5-8$, respectively. Patients with higher TBS-ALBI scores had decreased survival compared with lower TBS-ALBI scores in the derivation cohort (Figure $2 \mathrm{~A}, p<0.001$ ).
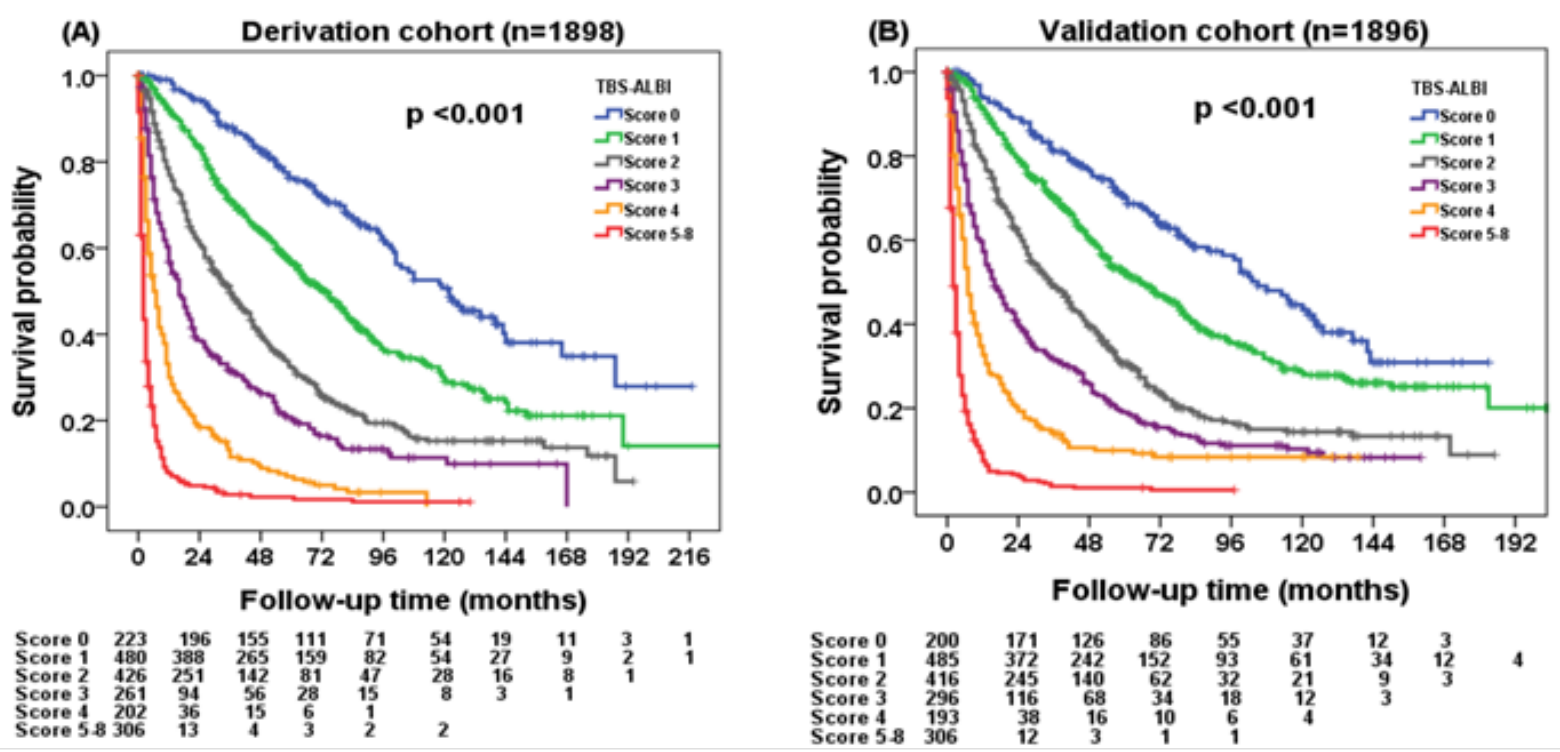

Figure 2. Kaplan-Meier analysis of the TBS- and ALBI-grade-based prognostic model (TBS-ALBI system) in the (A) derivation and (B) validation cohorts. There were significant survival differences in different TBS-ALBI score risk groups in the derivation cohort $(p<0.001 ; n=1898)$. Patients with high TBS-ALBI scores consistently had decreased overall survival in the validation cohort $(p<0.001$; $n=1896)$.

\subsection{Validation of the TBS-ALBI System}

Significant survival differences were found in different TBS-ALBI score groups of the validation cohort (Figure 2B, $p<0.001$ ). The 1-, 3-, and 5-year survival rates were 94\%, 81\%, 
and $68 \%$ for TBS-ALBI score $0,90 \%, 70 \%$, and $52 \%$ for score $1,76 \%, 47 \%$, and $29 \%$ for score $2,56 \%, 30 \%$, and $18 \%$ for score $3,32 \%, 14 \%$, and $11 \%$ for score 4 , and $8 \%, 2 \%$, and $2 \%$ for scores of 5-8, respectively.

The prognostic performance of the TBS-ALBI system was compared with other staging systems, including BCLC, HKLC, TIS, JIS, CLIP, Okuda, Tokyo, and TNM (Table 4). The TBS-ALBI system had the lowest AICc and the highest homogeneity, indicating better prognostic performance than other systems. In subgroup analysis stratified by curative $(n=902)$ and non-curative treatments $(n=994)$, the TBS-ALBI system still outperformed other staging systems. The TBS-ALBI system was consistently a better prognostic model when stratified by the etiology of $\operatorname{HBV}(n=776)$ and HCV $(n=374)$ groups.

Table 4. Prognostic performance of different staging systems in the validation cohort.

\begin{tabular}{|c|c|c|}
\hline Models & $\begin{array}{l}\text { Homogeneity } \\
\left(\text { Wald } \chi^{2}\right)\end{array}$ & $\begin{array}{c}\text { Corrected Akaike } \\
\text { Information Criterion }\end{array}$ \\
\hline \multicolumn{3}{|c|}{ Validation cohort $(n=1896)$} \\
\hline BCLC & 477.462 & $18,747.588$ \\
\hline HKLC & 543.712 & $18,681.338$ \\
\hline TIS & 676.704 & $18,544.279$ \\
\hline JIS & 503.527 & $18,721.523$ \\
\hline CLIP & 756.433 & $18,468.617$ \\
\hline Okuda & 466.310 & $18,758.439$ \\
\hline Tokyo & 517.941 & $18,707.108$ \\
\hline TNM & 294.309 & $18,930.740$ \\
\hline TBS-ALBI & 871.542 & $18,353.503$ \\
\hline \multicolumn{3}{|c|}{ Curative treatment $(n=902)$} \\
\hline BCLC & 35.453 & 6429.229 \\
\hline HKLC & 36.586 & 6428.089 \\
\hline TIS & 35.420 & 6427.088 \\
\hline JIS & 39.044 & 6425.638 \\
\hline CLIP & 51.544 & 6413.138 \\
\hline Okuda & 30.110 & 6434.517 \\
\hline Tokyo & 36.884 & 6427.798 \\
\hline TNM & 12.795 & 6451.887 \\
\hline TBS-ALBI & 76.604 & 6388.087 \\
\hline \multicolumn{3}{|c|}{ Non-curative treatment $(n=994)$} \\
\hline BCLC & 250.074 & $10,420.458$ \\
\hline HKLC & 268.381 & $10,402.051$ \\
\hline TIS & 380.781 & $10,289.651$ \\
\hline JIS & 241.444 & $10,428.988$ \\
\hline CLIP & 372.307 & $10,298.125$ \\
\hline Okuda & 205.407 & $10,465.025$ \\
\hline Tokyo & 198.510 & $10,471.922$ \\
\hline TNM & 162.579 & $10,507.853$ \\
\hline TBS-ALBI & 432.154 & $10,238.278$ \\
\hline \multicolumn{3}{|c|}{ HBV-related HCC $(n=776)$} \\
\hline BCLC & 201.496 & 6260.271 \\
\hline HKLC & 225.089 & 6235.670 \\
\hline TIS & 257.214 & 6202.857 \\
\hline JIS & 204.453 & 6257.314 \\
\hline CLIP & 278.184 & 6183.584 \\
\hline Okuda & 181.108 & 6280.659 \\
\hline Tokyo & 203.245 & 6258.522 \\
\hline TNM & 130.840 & 6330.928 \\
\hline TBS-ALBI & 359.468 & 6102.300 \\
\hline \multicolumn{3}{|c|}{ HCV-related HCC $(n=374)$} \\
\hline BCLC & 115.183 & 2894.521 \\
\hline HKLC & 135.930 & 2873.744 \\
\hline TIS & 144.346 & 2863.157 \\
\hline JIS & 105.382 & 2904.322 \\
\hline CLIP & 185.719 & 2823.985 \\
\hline Okuda & 85.960 & 2923.744 \\
\hline Tokyo & 97.339 & 2682.659 \\
\hline TNM & 47.638 & 2962.066 \\
\hline TBS-ALB & 189.422 & 2820.261 \\
\hline
\end{tabular}




\subsection{Using the TBS-ALBI System to Differentiate Survival in Different Risk Groups}

The discriminatory ability of survival for the TBS-ALBI system was evaluated in all HCC patients. The TBS-ALBI system can differentiate survival status well in either HBV-related or HCV-related HCC $(p<0.001$, Figure 3A,B). The survival difference was consistently identified in patients stratified by BCLC stage $0 / \mathrm{A}(n=1227)$ and stage $\mathrm{B} / \mathrm{C} / \mathrm{D}$ patients $(n=2567)(p<0.001$, Figure 4A,B). Patients with lower TBS-ALBI scores had better overall survival compared with higher TBS-ALBI scores stratified by curative $(n=1807)$ and non-curative treatments $(n=1987)(p<0.001$, Figure 5A,B).
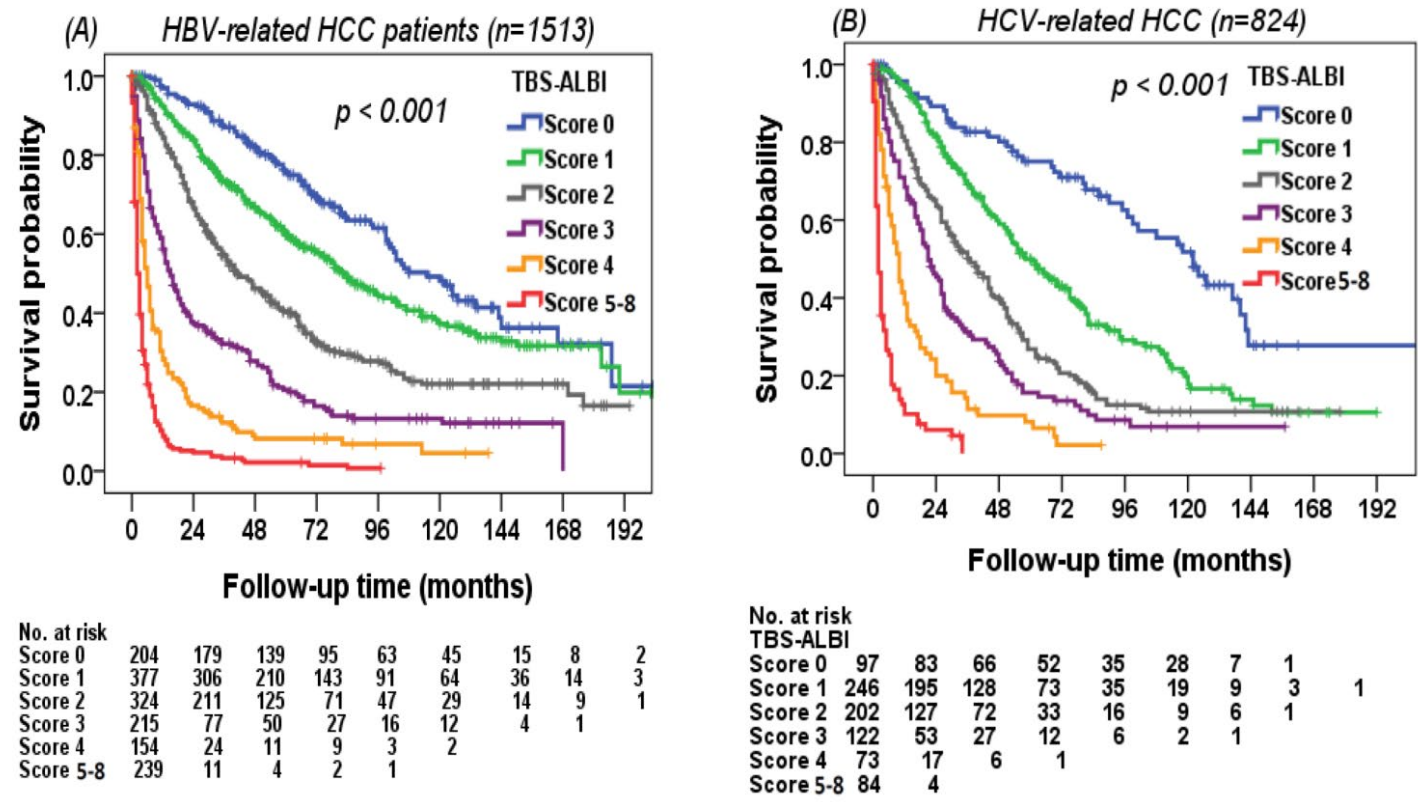

Figure 3. Survival distribution according to the TBS-ALBI system in (A) HBV-related HCC and (B) HCV-related HCC. Significant survival differences were found between different TBS-ALBI score groups in HBV-related $(p<0.001 ; n=1513)$ and HCV-related HCC $(p<0.001, n=824)$.
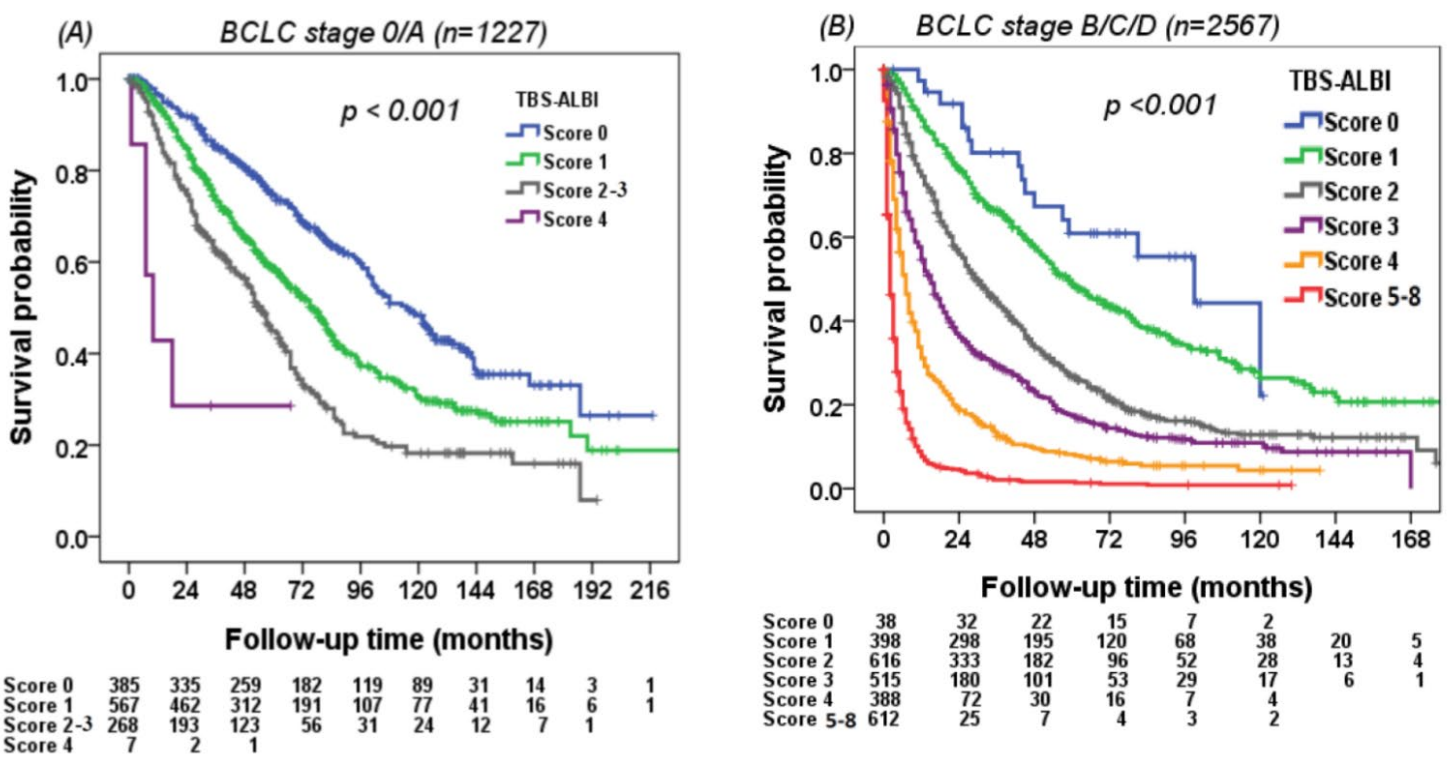

Figure 4. Survival distribution according to the TBS-ALBI system in (A) BCLC stage 0/A patients and (B) BCLC stage B-D patients. Patients with lower TBS-ALBI scores had better overall survival compared with those with higher scores in BCLC stage $0 / \mathrm{A}(p<0.001 ; n=1227)$ and BCLC stages B-D $(p<0.001, n=2567)$. 

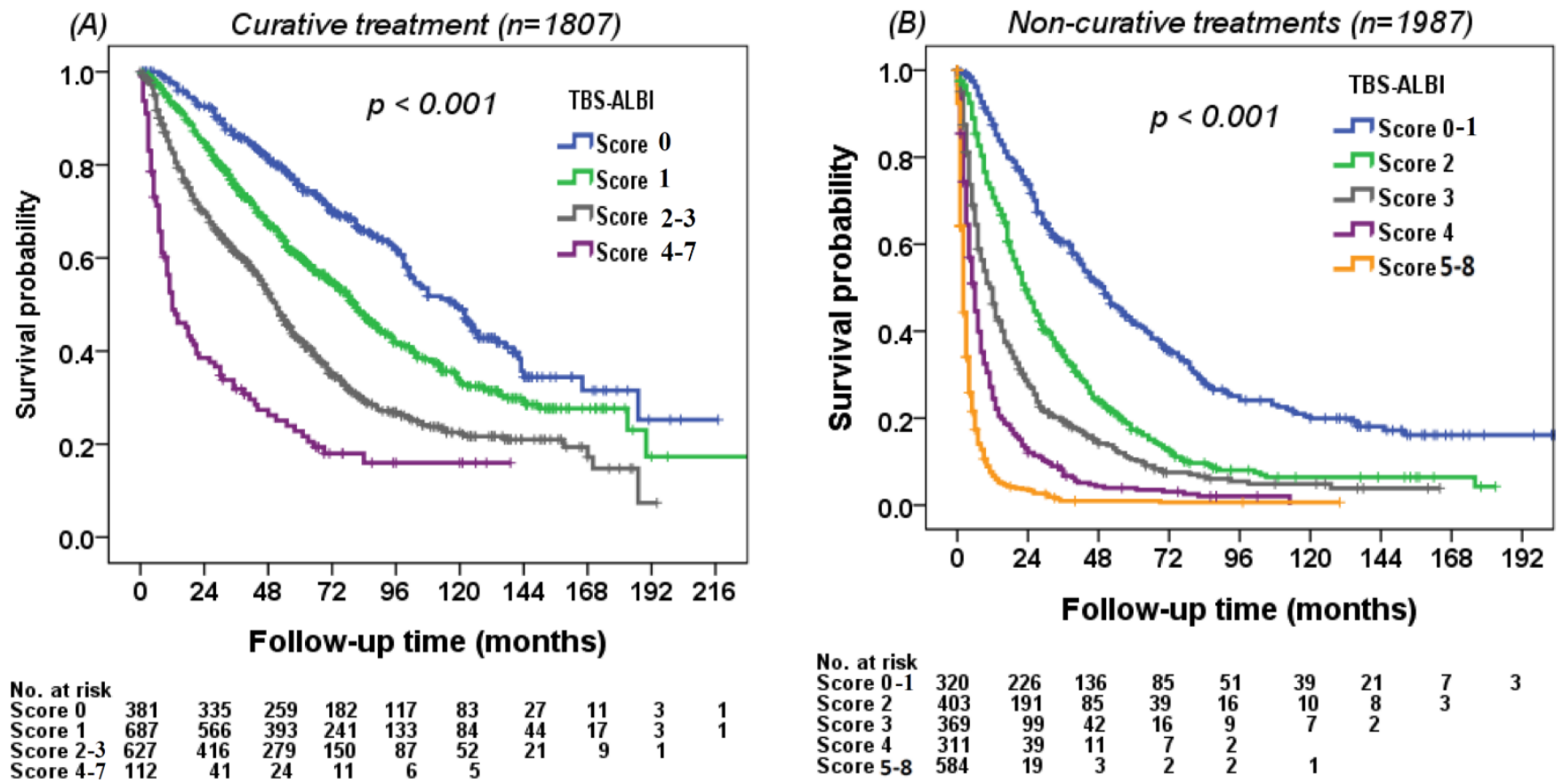

Figure 5. Survival distribution according to the TBS-ALBI system in patients receiving (A) curative and (B) non-curative treatments. Patients with high TBS-ALBI scores had increased risk of mortality compared with those with lower scores in patients receiving curative $(p<0.001 ; n=1807)$ and non-curative treatments $(p<0.001 ; n=1987)$.

\section{Discussion}

Staging systems provide crucial information in prognostic prediction for cancer patients. Quite a few staging systems have been proposed for outcome prediction in HCC, but the optimal model remains highly debatable. The BCLC staging system is currently the recommended system for HCC, and is included in the practice guidelines. However, a major drawback of the BCLC is that the outcomes are highly variable even for patients within the same stage $[13,30]$. In this study, we proposed and validated a new prognostic model-the TBS-ALBI system-for HCC. The TBS-ALBI system offers superior prognostic performance in comparison with other staging systems. In addition, its prognostic ability is stably consistent in patients with viral HCC. Notably, the TBS-ALBI score can discriminate overall survival well in patients with different BCLC stages and treatment strategies. Thus, the TBS-ALBI system is a very informative prognostic tool for HCC patients with different clinical characteristics.

Tumor burden, severity of liver dysfunction, and performance status are known important prognostic predictors for HCC. Typically, the size and number of tumor nodules are used to assess tumor burden, and have been included in many staging systems. These two variables are binary in nature, and could make it difficult to clearly differentiate the outcomes of HCC patients with variable tumor size and numbers. For example, the prognosis is quite different in patients with a single large HCC of $6 \mathrm{~cm}$ compared to those with three nodules of $4 \mathrm{~cm}, 3 \mathrm{~cm}$, and $2 \mathrm{~cm}$ in diameter. Thus, shifting from dichotomous to continuous variables of tumor size and number may enhance the prognostic ability. By using the Pythagorean theorem, TBS considers the collective impact of tumor diameter and number of tumors, encompassing the magnitude of tumor burden [12,15]. As such, TBS is a single and continuous variable to indicate tumor burden in HCC, as opposed to dichotomous variables such as the Milan criteria and Up-to-7 criteria. In addition, TBS is easy to calculate, requiring only maximal tumor diameter and number of nodules, as opposed to other calculation methods that require the diameter and number of all tumors. We found that patients with high TBS had a higher mortality risk than those with low and 
medium TBS in the multivariate Cox model. Consistent with previous studies [13-15,31,32], our study confirms that TBS is a feasible prognostic marker to assess tumor burden in HCC.

The management of HCC is associated with underlying liver dysfunction. The CTP classification and MELD score were designed for HCC patients with cirrhosis. However, these two models have potential limitations, because $20 \%$ of HCCs arise from non-cirrhotic livers. In this regard, the ALBI grade is a more objective marker of liver dysfunction, and can discriminate patient survival well, with high predictive accuracy [18-22]. In this study, ALBI grade 1 patients clearly had better survival compared with ALBI grade 2 and 3 patients in the multivariate Cox model. This finding consistently suggests that ALBI grade is a reliable prognostic tool to evaluate hepatic reserve in HCC.

Vascular invasion and distant metastasis typically indicate advanced cancer stages, and are often associated with large tumor burden in HCC [27,33]. In this study, patients with vascular invasion or metastasis had 2.3-fold increased risk of mortality compared with those without these features. Alternatively, ascites is a classical hallmark of portal hypertension [34]. The presence of ascites in HCC patients indicates not only poor hepatic functional reserve, but also aggressive tumor behavior [35]. In multivariate analysis, the presence of ascites was associated with a $34 \%$ increased risk of mortality compared with patients without ascites. Consistently with previous studies [35,36], we can confirm that ascites is an indispensable prognostic predictor for HCC.

AFP is a widely used biomarker for HCC in both diagnosis and prognostic prediction [37]. AFP is intimately associated with cell proliferation and cancer progression $[38,39]$. AFP also tightly coexists with large tumor burden, and predicts decreased survival in HCC patients [37]. Our data further suggest that AFP is an important parameter in prognostic prediction for HCC. Another meaningful finding in this study is our confirmation that performance status is a strong surrogate to indicate patient survival. Patients with a suboptimal performance status had a 1.8-fold increased risk of mortality compared with those with good performance status. Taken together, TBS, ALBI grade, ascites, vascular invasion or distant metastasis, AFP, and performance status are all crucial prognostic determinants for HCC. By using these six predictors, we developed and validated the novel TBS-ALBI system to predict long-term outcomes in HCC patients.

The TBS-ALBI system has several strengths. Firstly, TBS calculates tumor burden in a continuous fashion, and can stratify different risk groups to discriminate outcomes in HCC patients. Secondly, the ALBI grade is a simple and objective method to indicate hepatic functional reserve, with good prognostic performance in HCC patients, since most HCCs have mild fibrosis or cirrhosis at the time of diagnosis. Thirdly, the TBS-ALBI system is derived from clinical and objective laboratory variables, and is easy to calculate, without the need for complex computing to assess prognosis. Lastly, the TBS-ALBI system offers superior prognostic performance compared with other staging systems for HCC. Thus, the TBS-ALBI system could be a novel tool for making clinical decisions for patients with HCC.

There are nevertheless a few limitations to the present study. Firstly, the TBS-ALBI system was developed from a single center in Taiwan. External validation from different geographic regions is required in order to validate our results. Secondly, although TBS is a feasible marker to indicate tumor burden, the tumor diameter and number of nodules have the same prognostic statistical weight, which may require adjustment. Lastly, anticancer treatments are primarily decided based on shared decision making; as such, some patients may not strictly adhere to the BCLC recommendations.

\section{Conclusions}

The TBS-ALBI system, derived from six clinical predictors, is a novel, simple, and user-friendly prognostic model to evaluate HCC; its prognostic performance is better than that of other currently used staging systems. The predictive ability of the TBS-ALBI system remains consistently stable across different BCLC stages, treatments, and viral etiologies for 
outcome evaluation. External validation from different study groups is required in order to further validate our results.

Author Contributions: Conceptualization, T.-I.H.; data curation, S.-Y.H.; formal analysis, S.-Y.H.; funding acquisition, T.-I.H.; investigation, C.-Y.H., P.-H.L., J.-I.L. and T.-I.H.; methodology, C.-Y.H., Y.-H.H., C.-W.S., J.-I.L. and M.-C.H.; software, P.-H.L.; supervision, Y.-H.H. and M.-C.H.; validation, C.-W.S.; writing—original draft preparation, S.-Y.H.; writing—review and editing, T.-I.H. All authors have read and agreed to the published version of the manuscript.

Funding: This research was funded by Taipei Veterans General Hospital (grant number: V110C-001, VN111-07), Taipei, Taiwan.

Institutional Review Board Statement: This study was conducted according to the guidelines of the Declaration of Helsinki, and approved by the Institutional Review Board of Taipei Veterans General Hospital (protocol code: 2018-03-004CC; date of approval: 30 March 2018).

Informed Consent Statement: Informed consent was waived by the IRB due to the retrospective nature of this study.

Data Availability Statement: The data presented in this study are available on request from the corresponding author. The data are not publicly available due to ethical concerns.

Acknowledgments: We thank Chia-Lin Wang from Department of Medical Research, Taipei Veterans General Hospital, Taipei, Taiwan, in preparing this manuscript.

Conflicts of Interest: There authors declare no conflict of interest.

\section{References}

1. Bray, F.; Ferlay, J.; Soerjomataram, I.; Siegel, R.L.; Torre, L.A.; Jemal, A. Global cancer statistics 2018: GLOBOCAN estimates of incidence and mortality worldwide for 36 cancers in 185 countries. CA Cancer J. Clin. 2018, 68, 394-424. [CrossRef]

2. Heimbach, J.K.; Kulik, L.M.; Finn, R.S.; Sirlin, C.B.; Abecassis, M.M.; Roberts, L.R.; Zhu, A.X.; Murad, M.H.; Marrero, J.A. AASLD guidelines for the treatment of hepatocellular carcinoma. Hepatology 2018, 67, 358-380. [CrossRef]

3. European Association for the Study of the Liver. EASL Clinical Practice Guidelines: Management of hepatocellular carcinoma. J. Hepatol. 2018, 69, 182-236. [CrossRef]

4. Makary, M.S.; Khandpur, U.; Cloyd, J.M.; Mumtaz, K.; Dowell, J.D. Locoregional therapy approaches for hepatocellular carcinoma: Recent advances and management strategies. Cancers 2020, 12, 1914. [CrossRef]

5. Nakano, S.; Eso, Y.; Okada, H.; Takai, A.; Takahashi, K.; Seno, H. Recent advances in immunotherapy for hepatocellular carcinoma. Cancers 2020, 12, 775. [CrossRef]

6. Liu, P.H.; Hsu, C.Y.; Hsia, C.Y.; Lee, Y.H.; Su, C.W.; Huang, Y.H.; Lee, F.Y.; Lin, H.C.; Huo, T.I. Prognosis of hepatocellular carcinoma: Assessment of eleven staging systems. J. Hepatol. 2016, 64, 601-608. [CrossRef]

7. Mazzaferro, V.; Chun, Y.S.; Poon, R.T.; Schwartz, M.E.; Yao, F.Y.; Marsh, J.W.; Bhoori, S.; Lee, S.G. Liver transplantation for hepatocellular carcinoma. Ann. Surg. Oncol. 2008, 15, 1001-1007. [CrossRef]

8. Yasui, Y.; Tsuchiya, K.; Kurosaki, M.; Takeguchi, T.; Takeguchi, Y.; Okada, M.; Wang, W.; Kubota, Y.; Goto, T.; Komiyama, Y.; et al. Up-to-seven criteria as a useful predictor for tumor downstaging to within Milan criteria and Child-Pugh grade deterioration after initial conventional transarterial chemoembolization. Hepatol. Res. 2018, 48, 442-450. [CrossRef]

9. Mazzaferro, V.; Llovet, J.M.; Miceli, R.; Bhoori, S.; Schiavo, M.; Mariani, L.; Camerini, T.; Roayaie, S.; Schwartz, M.E.; Grazi, G.L.; et al. Predicting survival after liver transplantation in patients with hepatocellular carcinoma beyond the Milan criteria: A retrospective, exploratory analysis. Lancet Oncol. 2009, 10, 35-43. [CrossRef]

10. Lai, Q.; Avolio, A.W.; Manzia, T.M.; Sorge, R.; Agnes, S.; Tisone, G.; Berloco, P.B.; Rossi, M. Combination of biological and morphological parameters for the selection of patients with hepatocellular carcinoma waiting for liver transplantation. Clin. Transpl. 2012, 26, E125-E131. [CrossRef]

11. Hsu, C.Y.; Huang, Y.H.; Hsia, C.Y.; Su, C.W.; Lin, H.C.; Loong, C.C.; Chiou, Y.Y.; Chiang, J.H.; Lee, P.C.; Huo, T.I.; et al. A new prognostic model for hepatocellular carcinoma based on total tumor volume: The Taipei Integrated Scoring System. J. Hepatol. 2010, 53, 108-117. [CrossRef]

12. Sasaki, K.; Morioka, D.; Conci, S.; Margonis, G.A.; Sawada, Y.; Ruzzenente, A.; Kumamoto, T.; Iacono, C.; Andreatos, N.; Guglielmi, A.; et al. The Tumor Burden Score: A new "metro-ticket" prognostic tool for colorectal liver metastases based on tumor size and number of tumors. Ann. Surg. 2018, 267, 132-141. [CrossRef]

13. Tsilimigras, D.I.; Moris, D.; Hyer, J.M.; Bagante, F.; Sahara, K.; Moro, A.; Paredes, A.Z.; Mehta, R.; Ratti, F.; Marques, H.P.; et al. Hepatocellular carcinoma tumour burden score to stratify prognosis after resection. Br. J. Surg. 2020, 107, 854-864. [CrossRef]

14. Moris, D.; Shaw, B.I.; McElroy, L.; Barbas, A.S. Using Hepatocellular carcinoma tumor burden score to stratify prognosis after liver transplantation. Cancers 2020, 12, 3372. [CrossRef] 
15. Vitale, A.; Lai, Q.; Farinati, F.; Bucci, L.; Giannini, E.G.; Napoli, L.; Ciccarese, F.; Rapaccini, G.L.; Di Marco, M.; Caturelli, E.; et al. Utility of tumor burden score to stratify prognosis of patients with hepatocellular cancer: Results of 4759 Cases from ITA.LI.CA Study Group. J. Gastrointest. Surg. 2018, 22, 859-871. [CrossRef]

16. Kumada, T.; Toyoda, H.; Tada, T.; Yasuda, S.; Tanaka, J. Changes in background liver function in patients with hepatocellular carcinoma over 30 years: Comparison of Child-Pugh classification and albumin bilirubin grade. Liver Cancer 2020, 9, 518-528. [CrossRef]

17. Huo, T.I.; Lin, H.C.; Hsia, C.Y.; Wu, J.C.; Lee, P.C.; Chi, C.W.; Lee, S.D. The model for end-stage liver disease based cancer staging systems are better prognostic models for hepatocellular carcinoma: A prospective sequential survey. Am. J. Gastroenterol. 2007, 102, 1920-1930. [CrossRef]

18. Johnson, P.J.; Berhane, S.; Kagebayashi, C.; Satomura, S.; Teng, M.; Reeves, H.L.; O’Beirne, J.; Fox, R.; Skowronska, A.; Palmer, D.; et al. Assessment of liver function in patients with hepatocellular carcinoma: A new evidence-based approach-the ALBI grade. $J$. Clin. Oncol. 2015, 33, 550-558. [CrossRef]

19. Huo, T.I. ALBI grade as a new player in hepatocellular carcinoma. J. Chin. Med. Assoc. 2019, 82, 112. [CrossRef]

20. Ho, S.Y.; Hsu, C.Y.; Liu, P.H.; Hsia, C.Y.; Lei, H.J.; Huang, Y.H.; Ko, C.C.; Su, C.W.; Lee, R.C.; Hou, M.C.; et al. Albumin-bilirubin grade-based nomogram of the BCLC system for personalized prognostic prediction in hepatocellular carcinoma. Liver Int. 2020, 40, 205-214. [CrossRef]

21. Ho, S.Y.; Hsu, C.Y.; Liu, P.H.; Ko, C.C.; Huang, Y.H.; Su, C.W.; Lee, R.C.; Hou, M.C.; Huo, T.I. Survival of patients with hepatocellular carcinoma in renal insufficiency: Prognostic role of albumin-bilirubin grade. Cancers 2020, 12, 1130. [CrossRef]

22. Lescure, C.; Estrade, F.; Pedrono, M.; Campillo-Gimenez, B.; Le Sourd, S.; Pracht, M.; Palard, X.; Bourien, H.; Muzellec, L.; Uguen, T.; et al. ALBI score is a strong predictor of toxicity following SIRT for hepatocellular carcinoma. Cancers 2021, 13, 3794. [CrossRef]

23. Liu, P.H.; Lee, Y.H.; Hsia, C.Y.; Hsu, C.Y.; Huang, Y.H.; Chiou, Y.Y.; Lin, H.C.; Huo, T.I. Surgical resection versus transarterial chemoembolization for hepatocellular carcinoma with portal vein tumor thrombosis: A propensity score analysis. Ann. Surg. Oncol. 2014, 21, 1825-1833. [CrossRef]

24. Huo, T.; Huang, Y.H.; Wu, J.C.; Chiang, J.H.; Lee, P.C.; Chang, F.Y.; Lee, S.D. Comparison of transarterial chemoembolization and percutaneous acetic acid injection as the primary loco-regional therapy for unresectable hepatocellular carcinoma: A prospective survey. Aliment. Pharmacol. 2004, 19, 1301-1308. [CrossRef]

25. Hsu, C.Y.; Hsia, C.Y.; Huang, Y.H.; Su, C.W.; Lin, H.C.; Pai, J.T.; Loong, C.C.; Chiou, Y.Y.; Lee, R.C.; Lee, F.Y.; et al. Comparison of surgical resection and transarterial chemoembolization for hepatocellular carcinoma beyond the Milan criteria: A propensity score analysis. Ann. Surg. Oncol. 2012, 19, 842-849. [CrossRef]

26. Liu, P.H.; Huo, T.I.; Miksad, R.A. Hepatocellular carcinoma with portal vein tumor involvement: Best management strategies. Semin. Liver Dis. 2018, 38, 242-251. [CrossRef]

27. Hsu, C.Y.; Liu, P.H.; Ho, S.Y.; Huang, Y.H.; Lee, Y.H.; Lee, R.C.; Nagaria, T.S.; Hou, M.C.; Huo, T.I. Metastasis in patients with hepatocellular carcinoma: Prevalence, determinants, prognostic impact and ability to improve the Barcelona Clinic Liver Cancer system. Liver Int. 2018, 38, 1803-1811. [CrossRef]

28. Hsu, C.Y.; Lee, Y.H.; Hsia, C.Y.; Huang, Y.H.; Su, C.W.; Lin, H.C.; Lee, R.C.; Chiou, Y.Y.; Lee, F.Y.; Huo, T.I. Performance status in patients with hepatocellular carcinoma: Determinants, prognostic impact, and ability to improve the Barcelona Clinic Liver Cancer system. Hepatology 2013, 57, 112-119. [CrossRef]

29. Pourhoseingholi, M.A.; Hajizadeh, E.; Moghimi Dehkordi, B.; Safaee, A.; Abadi, A.; Zali, M.R. Comparing Cox regression and parametric models for survival of patients with gastric carcinoma. Asian Pac. J. Cancer Prev. 2007, 8, 412-416.

30. Fang, K.C.; Kao, W.Y.; Su, C.W.; Chen, P.C.; Lee, P.C.; Huang, Y.H.; Huo, T.I.; Chang, C.C.; Hou, M.C.; Lin, H.C.; et al. The prognosis of single large hepatocellular carcinoma was distinct from Barcelona Clinic Liver Cancer Stage A or B: The role of albumin-bilirubin grade. Liver Cancer 2018, 7, 335-358. [CrossRef]

31. Tsilimigras, D.I.; Mehta, R.; Paredes, A.Z.; Moris, D.; Sahara, K.; Bagante, F.; Ratti, F.; Marques, H.P.; Silva, S.; Soubrane, O.; et al Overall tumor burden dictates outcomes for patients undergoing resection of multinodular hepatocellular carcinoma beyond the Milan criteria. Ann. Surg. 2020, 272, 574-581. [CrossRef] [PubMed]

32. Ho, S.Y.; Liu, P.H.; Hsu, C.Y.; Ko, C.C.; Huang, Y.H.; Su, C.W.; Lee, R.C.; Tsai, P.H.; Hou, M.C.; Huo, T.I. Tumor burden score as a new prognostic marker for patients with hepatocellular carcinoma undergoing transarterial chemoembolization. J. Gastroenterol. Hepatol. 2021, 36, 3196-3203. [CrossRef] [PubMed]

33. Lee, Y.H.; Hsu, C.Y.; Huang, Y.H.; Hsia, C.Y.; Chiou, Y.Y.; Su, C.W.; Lin, H.C.; Huo, T.I. Vascular invasion in hepatocellular carcinoma: Prevalence, determinants and prognostic impact. J. Clin. Gastroenterol. 2014, 48, 734-741. [CrossRef] [PubMed]

34. Aithal, G.P.; Palaniyappan, N.; China, L.; Härmälä, S.; Macken, L.; Ryan, J.M.; Wilkes, E.A.; Moore, K.; Leithead, J.A.; Hayes, P.C.; et al. Guidelines on the management of ascites in cirrhosis. Gut 2021, 70, 9-29. [CrossRef] [PubMed]

35. Hsu, C.Y.; Lee, Y.H.; Huang, Y.H.; Hsia, C.Y.; Su, C.W.; Lin, H.C.; Lee, R.C.; Chiou, Y.Y.; Lee, F.Y.; Huo, T.I.; et al. Ascites in patients with hepatocellular carcinoma: Prevalence, associated factors, prognostic impact, and staging strategy. Hepatol. Int. 2013, 7, 188-198. [CrossRef]

36. Ho, S.Y.; Liu, P.H.; Hsu, C.Y.; Hsia, C.Y.; Huang, Y.H.; Su, C.W.; Lei, H.J.; Lee, R.C.; Hou, M.C.; Huo, T.I. A new prognostic model based on albumin-bilirubin grade for hepatocellular carcinoma beyond the Milan criteria. Dig. Dis. Sci. 2020, 65, 658-667. [CrossRef] 
37. Hsu, C.Y.; Liu, P.H.; Lee, Y.H.; Hsia, C.Y.; Huang, Y.H.; Lin, H.C.; Chiou, Y.Y.; Lee, F.Y.; Huo, T.I. Using serum alpha-fetoprotein for prognostic prediction in patients with hepatocellular carcinoma: What is the most optimal cutoff? PLoS ONE 2015, 10, e0118825

38. Galle, P.R.; Foerster, F.; Kudo, M.; Chan, S.L.; Llovet, J.M.; Qin, S.; Schelman, W.R.; Chintharlapalli, S.; Abada, P.B.; Sherman, M.; et al. Biology and significance of alpha-fetoprotein in hepatocellular carcinoma. Liver Int. 2019, 39, 2214-2229. [CrossRef]

39. Cassim, S.; Raymond, V.A.; Dehbidi-Assadzadeh, L.; Lapierre, P.; Bilodeau, M. Metabolic reprogramming enables hepatocarcinoma cells to efficiently adapt and survive to a nutrient-restricted microenvironment. Cell Cycle 2018, 17, 903-916. [CrossRef] 